

\section{ABSTRACTS OF THESES}

David A. Klarner, Ph. D., Enumeration involving Sums over Compositions, University of Alberta, Edmonton, September 1966. (Supervisor: E. L. Whitney (deceased))

In this thesis we have presented a theory for sums having the form

$$
b(n)=\Sigma f\left(a_{1}, a_{2}\right) f\left(a_{2}, a_{3}\right) \ldots f\left(a_{i-1}, a_{i}\right) g\left(a_{i}\right)
$$

where the sum extends over all compositions $\left(a_{1}, a_{2}, \ldots, a_{i}\right)$ of $n$ into an unrestricted number of positive parts. The numbers $\{f(m, n)\}$ and $\{\mathrm{g}(\mathrm{n})\}$ are given by generating functions $\mathrm{F}(\mathrm{x}, \mathrm{y})$ and $\mathrm{G}(\mathrm{x})$ respectively, and we seek a relationship between these functions and the generating function $B(x, y)=\Sigma b(a, n) y^{a} x^{n}$, where $b(a, n)$ denotes $\mathrm{n}, \mathrm{a}$

the partial sum obtained from (1) by summing over just those compositions of $\mathrm{n}$ with $\mathrm{a}_{1}=\mathrm{a}$. We show in Chapter $\mathrm{I}$ that

$$
B(x, y)=G(x y)+\frac{1}{2 \pi i} \int_{C} F\left(x y, \frac{1}{s}\right) B(x, s) \frac{d s}{s},
$$

where $\mathrm{c}$ is a contour in the $\mathrm{s}$ plane which includes the singularities of $F\left(x y, \frac{1}{s}\right) / s$, but excludes those of $B(x, s)$. The relation (2) is a special case of the Fredholm integral equation which can be solved by standard methods described in Chapter I.

Chapter II begins with a concise definition of an n-omino and a description of the waxk done on the cell growth problem. Eden proved that the following special case of (1) gives a lower bound for the number of $n$-ominoes:

$$
b(n)=\Sigma\left(a_{1}+a_{2}-1\right)\left(a_{2}+a_{3}-1\right) \ldots\left(a_{i-1}+a_{i}-1\right) .
$$

Using the methods described in Chapter I, we find the generating function of $\{b(n)\}$ as defined by (3); also, we find this generating function by an elementary method in order to show, in one instance at least, the advantage of treating the Fredholm equation.

In Chapter III we prove there are more than $(3.6)^{\mathrm{n}}$ n-ominoes for all sufficiently large $n$. To do this we prove that

$$
f\left(a_{1}, a_{2}, \ldots, a_{i}\right) \geq f\left(a_{1}, a_{2}\right) f\left(a_{2}, a_{3}\right) \ldots f\left(a_{i-1}, a_{i}\right),
$$


where $\left(a_{1}, a_{2}, \ldots, a_{i}\right)$ denotes any composition of $n$, and $f(p, q, \ldots)$ denotes the number of $n$-ominoes with $p$ cells in the first row, $q$ cells in the second row, and so on. Next we find the generating function of $\{\mathrm{f}(\mathrm{m}, \mathrm{n})\}$, and use the Fredholm equation to find a lower bound for

$$
b(n)=\Sigma f\left(a_{1}, a_{2}\right) f\left(a_{2}, a_{3}\right) \ldots f\left(a_{i-1}, a_{i}\right)
$$

which is in turn a lower bound for the number of n-ominoes.

Read stated and proved a general combinatorial theorem which he used to find the number of n-ominoes with $1,2,3,4$, or 5 rows of cells. In Chapter IV we solve Read's problem by means of the Fredholm equation. Also, we indicate a method for finding the number of $n$-ominoes with $k$ rows using linear difference equations.

Harary, Prins, and Tutte used Polya's theorem to enumerate planted plane trees; also, they gave a one-one correspondence between the planted plane trees with $n+2$ nodes and the (tri-valent) planted plane trees with $2 \mathrm{n}+2$ nodes in which each node has valence either 1 or 3. In Chapter $\mathrm{V}$ we show that the methods of Chapter $I$ apply to the se enumeration problems as well, and go on to enumerate special classes of planted plane trees, for example, planted planes trees having nodes with valance either 1 or $k$. Furthermore, we give a very simple correspondence between the planted plane trees and the tri-valent planted plane trees by matching these trees with the elements of a certain set of binary codes.

We discuss the connection between the Fredholm equation and the generating functions for certain arithmetic functions in Chapter VI. Sums extended over the divisors of $n$ may be thought of as sums extended over just those compositions of $n$ in which all of the parts are the same. Also, by defining

$$
f(m, n)=\left\{\begin{array}{cc}
f *(m, n), & \text { if } m \leq n \\
0, & \text { otherwise }
\end{array},\right.
$$

the index of summation in (1) is actually restricted to the partitions $\left(a_{1} \leq a_{2} \leq \cdots \leq a_{i}\right)$ of $n$, since all other compositions of $n$ only contribute 0 to the sum. Using this idea, we are able to establish a link between the Fredholm equation and the theory of partitions.

Chapter VII contains still another application of Pólya's fundamental theorem, this time to find the number of non-isomorphic generalized graphs with $n$ nodes. A generalized graph is a pair $\left(N_{n}, N\right)$, where $N_{n}=\{1,2, \ldots, n\}$ is the set of nodes, and $N$ is some subset of the $k$-tuples $\mathrm{N}_{n} \times \mathrm{N}_{\mathrm{n}} \times \ldots \times \mathrm{N}_{\mathrm{n}}$ called the edge set of the graph. 